\title{
A Dynamic Health Assessment Approach for Shearer Based on Artificial Immune Algorithm
}

\author{
Zhongbin Wang, ${ }^{1}$ Xihua Xu, ${ }^{1}$ Lei Si, ${ }^{1,2}$ Rui Ji, ${ }^{1}$ Xinhua Liu, ${ }^{1}$ and Chao $\operatorname{Tan}^{1,3}$ \\ ${ }^{1}$ School of Mechatronic Engineering, China University of Mining and Technology, Xuzhou 221116, China \\ ${ }^{2}$ School of Information and Electrical Engineering, China University of Mining and Technology, Xuzhou 221116, China \\ ${ }^{3}$ Xuyi Mine Equipment and Materials Re D Center, China University of Mining and Technology, Huaian 223001, China \\ Correspondence should be addressed to Xihua Xu; xuxihua_cumt@163.com
}

Received 25 January 2016; Revised 3 March 2016; Accepted 7 March 2016

Academic Editor: Cheng-Jian Lin

Copyright (C) 2016 Zhongbin Wang et al. This is an open access article distributed under the Creative Commons Attribution License, which permits unrestricted use, distribution, and reproduction in any medium, provided the original work is properly cited.

\begin{abstract}
In order to accurately identify the dynamic health of shearer, reducing operating trouble and production accident of shearer and improving coal production efficiency further, a dynamic health assessment approach for shearer based on artificial immune algorithm was proposed. The key technologies such as system framework, selecting the indicators for shearer dynamic health assessment, and health assessment model were provided, and the flowchart of the proposed approach was designed. A simulation example, with an accuracy of $96 \%$, based on the collected data from industrial production scene was provided. Furthermore, the comparison demonstrated that the proposed method exhibited higher classification accuracy than the classifiers based on back propagation-neural network (BP-NN) and support vector machine (SVM) methods. Finally, the proposed approach was applied in an engineering problem of shearer dynamic health assessment. The industrial application results showed that the paper research achievements could be used combining with shearer automation control system in fully mechanized coal face. The simulation and the application results indicated that the proposed method was feasible and outperforming others.
\end{abstract}

\section{Introduction}

Due to the randomicity and complexity of underground geological conditions, assessment of shearer health condition would present the characteristics of complexity, fuzziness, and uncertainty, and this may affect the coal production or even endanger the operator's life. Moreover, because of the poor mining environment and complex component structure of shearer, the shearer operator cannot accurately estimate the working status of shearer, which may lead to some problems of poor coal quality and low mining efficiency. Furthermore, an increasing number of safety accidents in collieries are caused frequently. Therefore, it is necessary to assess the dynamic health condition of shearer which has become a challenging and significant research subject [1].

Depending on the assessment of the health condition of shearer, this can reduce operating trouble and production accident of shearer and improve production efficiency further. In recent years, many researches have brought out some achievement on shearer health condition diagnosis.
The multiple fault classifier based on the improved support vector machine theory is used to judge the fault types of coal shearer [2]. In [3], a correct and timely diagnosis mechanism of shearer failures by knowledge acquisition through a fuzzy inference system is provided, which can approximate expert experience. Although many research achievements have been proposed, they have some common shortcomings summarized as follows. Firstly, most research cannot confirm the health degree clearly. Moreover, it costs long diagnosis time and cannot be used in real-time health assessment.

Dynamic health assessment was used in spacecraft primarily in the 1970s. At present, domestic and abroad researchers have worked on the modeling approaches for dynamic health assessment and proposed several solutions. The densitybased spatial clustering of applications with noise has been used for bearings' condition monitoring [4], and a novel online method based on dynamic Bayesian networks (DBNs) for the estimation of the SOH of lithium- (Li-) ion batteries has been presented [5], and so on. However, due to 
the complex component structure and bad working condition, there has not been a health assessment concept on shearer, and this paper tries to present it. In the real mining condition, some key index parameters have a strong relationship with shearer health condition. The relationship is highly nonlinear in nature so that it is hard to develop a comprehensive mathematic model. The current methods and mature assessment systems are hardly satisfied with the shearer health state assessment. In this paper, we try to propose a novel prediction approach for shearer dynamic health assessment to identify the health state during coal mining.

The first mathematical model in artificial immune system was proposed in 1974, which initiated subsequent researches and discussions. Artificial immune system (AIS), as a novel intelligent algorithm method, inspired from the biological immune system, is an effective means for prediction [68]. The AIS can acquire learning capability by learning the biological protection principle. According to the above analysis, a novel prediction approach for shearer dynamic health assessment based on artificial immune algorithm is proposed and the assessment system is validated by the sample data of operating parameters from industrial production scene. Moreover, it will prove that artificial immune algorithm is a better tool for classifying due to its classification accuracy than the classifiers based on back propagationneural network (BP-NN) and support vector machine (SVM) methods later.

The remainder of this paper is organized as follows. Some related works are outlined in Section 2. The key technologies such as system framework, selecting the indicators for shearer dynamic health assessment, and the proposed approach are presented in Section 3. Section 4 provides a simulation example and an industrial application example for shearer dynamic health assessment based on the proposed approach to specify the application effect. Our conclusions are summarized in Section 5.

\section{Literature Review}

Recent publications relevant to this paper are mainly concerned with two research streams: the dynamic health assessment methods and artificial immune algorithm. In this section, we try to summarize the relevant literatures.

2.1. The Dynamic Health Assessment Methods. For the dynamic health assessment problem, lots of research has been done since the last decades. In [9], Zhong-Bin et al. developed a remote monitoring platform of the shearer by using VirtualPrototype technology to realize the remote monitoring for the shearer in the fully mechanized long-wall coal mining face. In [10], Zhou et al. proposed a novel approach based on the coal floor height variation which is taken as a significant factor and fuzzy optimization theory to improve the implement precision of shearer memory cutting. In [11], P. W. Tse and Y. L. Tse designed an innovative system that is installed in a passenger car or a truck that is running on road and provides instantaneous engine health evaluation and diagnosis. In [12], Black and Winiewicz provided a method and apparatus for internal network device dynamic health monitoring to increase network device availability. In [13], Vichare and Pecht presented the state of practice and the current state of research in the area of electronics prognostics and health management. In [14], Pecht and Jaai presented an assessment of the state of practice in prognostics and health management of information and electronics-rich systems. In [15], Yang et al. proposed an accurate identification of the shearer late underground cutting coal and rock conditions and fault diagnosis by the method of vibration analysis. In [16], Yin et al. designed an embedded health evaluation system to meet the requirement of continuous monitoring of the mine special gear box. In [17], Mascareñas et al. investigated a vibrohaptic human-machine interface for structural health monitoring. In [18], Cerda et al. explored an indirect approach for structural health bridge monitoring allowing for wide, yet cost-effective, bridge stock coverage. In [19], Zubizarreta-Rodriguez and Vasudevan introduced a new multisensor measurement framework for condition monitoring of brushless DC motors (BLDCM) with bearings. In [4], Kerroumi et al. introduced a dynamic classification method inspired by DBSCAN clustering method for machine condition monitoring in general and for bearings in particular. In [5], He et al. presented a novel online method for the estimation of the $\mathrm{SOH}$ of lithium- (Li) ion batteries based on dynamic Bayesian networks (DBNs). In [20], Herrmann et al. gave an introduction into the principle of structural health monitoring (SHM), basics of fatigue of fiber resin composite materials, and the possible application of these principles in the automotive industry.

2.2. Artificial Immune Algorithm. The artificial immune algorithm was firstly proposed by Farmer in 1986 [21]. It is able to recognize novel shapes without preprogramming based on the capacity of learning, memory, and pattern recognition. In [22], Ishiguro et al. proposed a new decentralized consensusmaking system inspired from the biological immune system and an adaptation mechanism that can be used to construct a suitable immune network for adequate action selection. In [23], Tang et al. described a new model of multiple-valued immune network based on biological immune response network. In [24], Abbattista et al. proposed the use of immune network model for designing associative memories. In [25], Deng et al. proposed a fuzzy logic resource allocation and memory cell pruning based artificial immune recognition system (AIRS) to improve the resource allocation mechanism of AIRS and decrease the memory cells. In [26], De Castro and Von Zuben proposed computational implementation of the clonal selection principle that explicitly takes into account the affinity maturation of the immune response. In [27], Chun et al. presented a new method employing the immune algorithm (IA) as the search method for the shape optimization of an electromagnetic device. In [28], Endoh et al. proposed an optimization algorithm based on immune model and applied it to the $n$th agents' travelling salesman problem called $n$-TSP. In [29], Ishiguro et al. proposed a new inference/consensus-making system inspired by immune systems in living organisms, and they apply 
the proposed method to the behavior arbitration of an autonomous mobile robot as a practical example. In [30], Harmer et al. developed a self-adaptive distributed agentbased defense immune system based on biological strategies within a hierarchical layered architecture. In [31], Pan et al. presented an immune dominance clonal selection multiobjective algorithm based on the artificial immune system to further improve the performance of the optimization algorithm for locomotive secondary spring load adjustment. In [32], Souza et al. presented two new approaches to solving the reconfiguration problem of electrical distribution systems (EDS) using the Copt-aiNet (Artificial Immune Network for Combinatorial Optimization) and Opt-aiNet (Artificial Immune Network for Optimization) algorithms. In [33], Zhang et al. proposed a novel fuzzy hybrid quantum artificial immune clustering algorithm based on cloud model (CFHQAI) to solve the stochastic problem. In [34], Savsani et al. presented the effect of hybridizing BiogeographyBased Optimization (BBO) technique with artificial immune algorithm (AIA) and Ant Colony Optimization (ACO) in two different ways. In [35], Kuo et al. were dedicated to proposing a cluster analysis algorithm which is integration of artificial immune network (aiNet) and $K$-means algorithm (aiNet $K$ ).

2.3. Discussion. According to the above researches, many health assessment methods, such as density-based spatial clustering and dynamic Bayesian networks, have been applied in the bearings' condition monitoring, network device dynamic health monitoring, and so on. But there are still no relevant studies on the dynamic health assessment methods for shearer. Considering the superiority and universality of artificial immune algorithm, this paper prepares to use this AI algorithm to predict the dynamic health status of shearer. A simulation experiment and an application example are carried out and the proposed approach is proved to be feasible and efficient.

\section{The Dynamic Health Assessment Approach Based on Artificial Immune Algorithm}

3.1. The Framework of the Proposed Approach. Some realtime running indicators of shearer are usually used to classify the health condition of shearer since the signals can describe its dynamic characteristics. In order to identify the dynamic health status of shearer, the following three processes are required. These processes are assessment indicators selecting, data acquisition and initialization, and multiclass classifiers training and testing. The proposed condition classification approach for shearer dynamic health state is shown in Figure 1 . The approach mainly consists of three critical steps: indicators selecting, data initialization, and data training and classification. Firstly, choosing the most effective indicators to assess the health condition of shearer is important since excessive assessment indicators will reduce the impact of main indicators and cause an incorrect result. Then, all the object data in the schema object set are normalized, so the attribute value is within the unit interval $[0,1]$ and the sample data are divided into four types. Finally, the artificial immune algorithm is used to classify the dynamic health status of shearer.

3.2. Selecting the Assessment Indicators. The system of shearer is made up by many subsystems. Establishing a scientific and reasonable evaluation system is the foundation of the health state evaluation for shearer. Depending on the actual operation situation of shearer and referencing other health assessment systems, the assessment consequences for shearer health can be divided into four typical modes: normal mode, transition mode, abnormal mode, and danger mode. The definition of each type of operation is given as follows.

Normal Mode. During the working process, the health indicators of shearer change a little and are all in normal range. The shearer works normally.

Transition Mode. During the working process, one or two health indicators of shearer have a wide range change occasionally and are not up to the danger line. The shearer works normally and meanwhile the worker of shearer must discover the problem and solve it.

Abnormal Mode. During the working process, some of the health indicators of shearer have a wide range change persistently and are not up to the danger line. The worker of shearer should stop coal production before returning it to normal.

Dangerous Mode. During the working process, some of the health indicators of shearer have a sudden change and are up to the danger line. The worker of shearer should stop coal production immediately.

By setting malfunction threshold value depending on operation situation, four modes of shearer health situation decrease progressively. Four different health modes can guide coal worker adopting corresponding operation, respectively.

The system of shearer is made up by many subsystems. The data from historical recording and real-time monitoring of the subsystems reflect the health status of shearer more or less. However, in practical application, we must choose the most effective indicators to assess the health situation of shearer and eliminate subordinate indicators, as excessive assessment indicators will reduce the impact of the main indicators, causing an incorrect result. According to the expert experience and actual working condition of shearer, the dynamic health condition depends on the real-time monitoring data. In this paper, the key content is the realtime health assessment of shearer. Thus, to assess the dynamic health situation of shearer, we choose nine real-time running indicators: the pulling speed $p_{1}$, the right cutting motor current $p_{2}$ and the left cutting motor current $p_{3}$, the right pulling motor current $p_{4}$ and the left pulling motor current $p_{5}$, the right cutting motor temperature $p_{6}$ and the left cutting motor temperature $p_{7}$, and the right pulling motor temperature $p_{8}$ and the left pulling motor temperature $p_{9}$. There are test data showing that the pulling speed has a mapping relation with working load of shearer. Monitoring the change of the pulling speed can reflect the working load in a degree. Moreover, as the most important information on judging shearer operating 


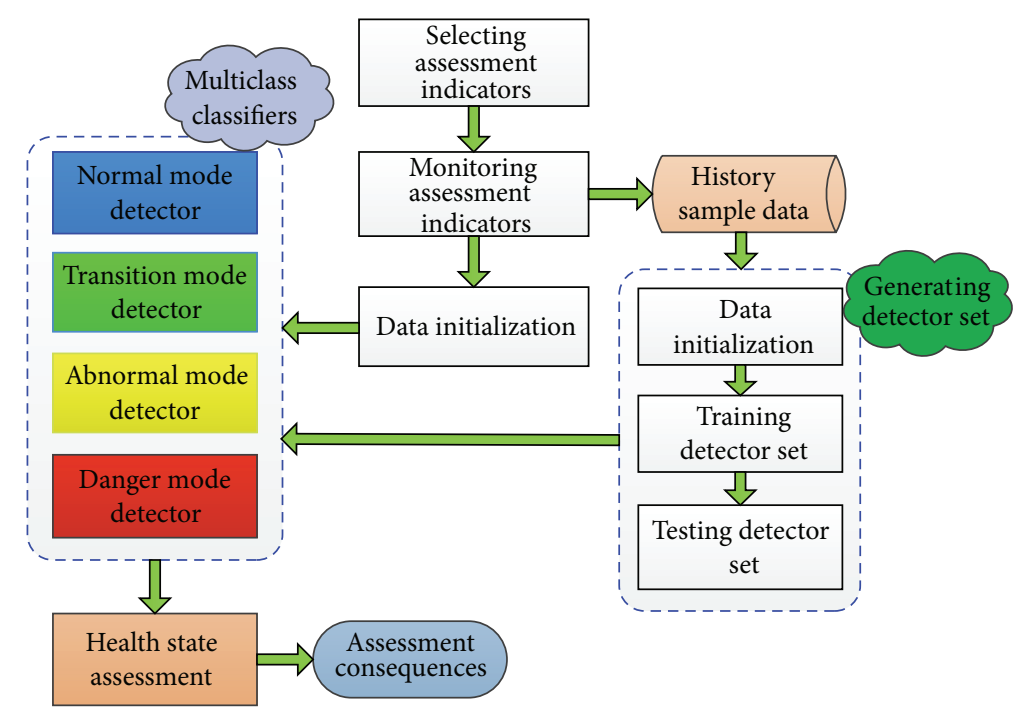

FIGURE 1: The framework of the proposed approach.

state, the cutting motor current and the pulling motor current can be influenced by the pulling speed, the cutting drum height, the working load, the coal-rock characteristic, and so on. The difference between the two currents is that the cutting motor current has a direct proportion with working load, while the pulling motor current can comprehensively characterize the pulling resistance. Finally, the slow change of the cutting motor temperature and the pulling motor temperature can represent the general state of working load and pulling load over a period of time. The nine indicators can reflect the shearer operation state clearly. The assessment indicators of dynamic health assessment model for shearer can be shown in Figure 2.

3.3. Defining Detectors Set. According to the nine indicators of shearer dynamic health assessment approach defined above, we can determine the unknown schema object $p^{\prime}$ for nine-dimensional attribute space, shown in detail in Definition 1. Corresponding to the four modes of shearer dynamic health assessment consequences, multiclass classifiers are constituted by four detectors: normal mode $\left(C_{1}\right)$, transition mode $\left(C_{2}\right)$, abnormal mode $\left(C_{3}\right)$, and danger mode $\left(C_{4}\right)$. Any one of the non-self-class objects (the schema object of classes $C_{1}, \ldots, C_{i-1}, C_{i+1}, \ldots, C_{4}$ ) can be recognized by the $i$ th detector $\left(R_{i}\right)$, excepting the self-class object (the schema object of class $C_{i}$ ). In other words, each detector only cannot recognize the corresponding class object of particular assessment consequence mode. The immune classifier model of dynamic health assessment for shearer is shown in Figure 3.

Before establishing the dynamic health assessment model based on artificial immune algorithm, the related definitions of multiclass classifiers are given as follows.

Definition 1. Each schema object can be represented as a $k$ dimensional vector $(p, c)=\left(p_{1}, p_{2}, \ldots, p_{k}, c\right)$, and $P$ is data

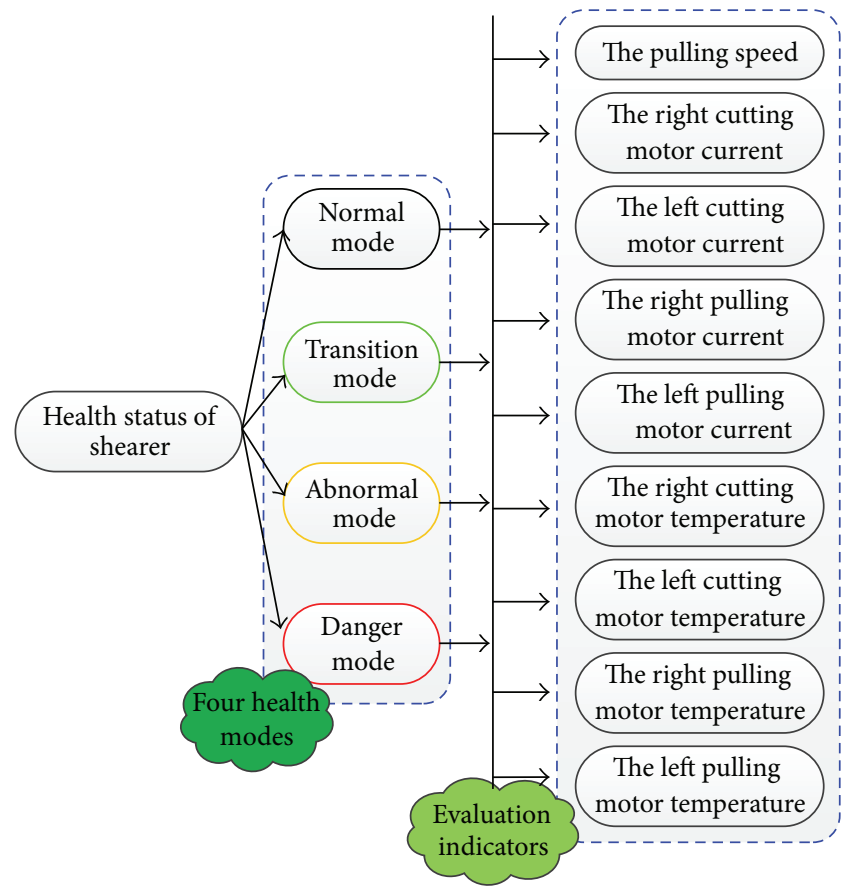

Figure 2: The indicators of dynamic health assessment model for shearer.

set of the schema object, $p \in P$, where $k=9$ is the number of attributes of the schema object and $c$ is attribute class of schema object.

Definition 2. Detectors set $R$ can recognize all the certain type data in the schema object set. Each member of the detectors set is called receptor, marked as $r,(r, c)=\left(r_{1}, r_{2}, \ldots, r_{k}, c\right)$. The receptor has similar structure to the schema object. 


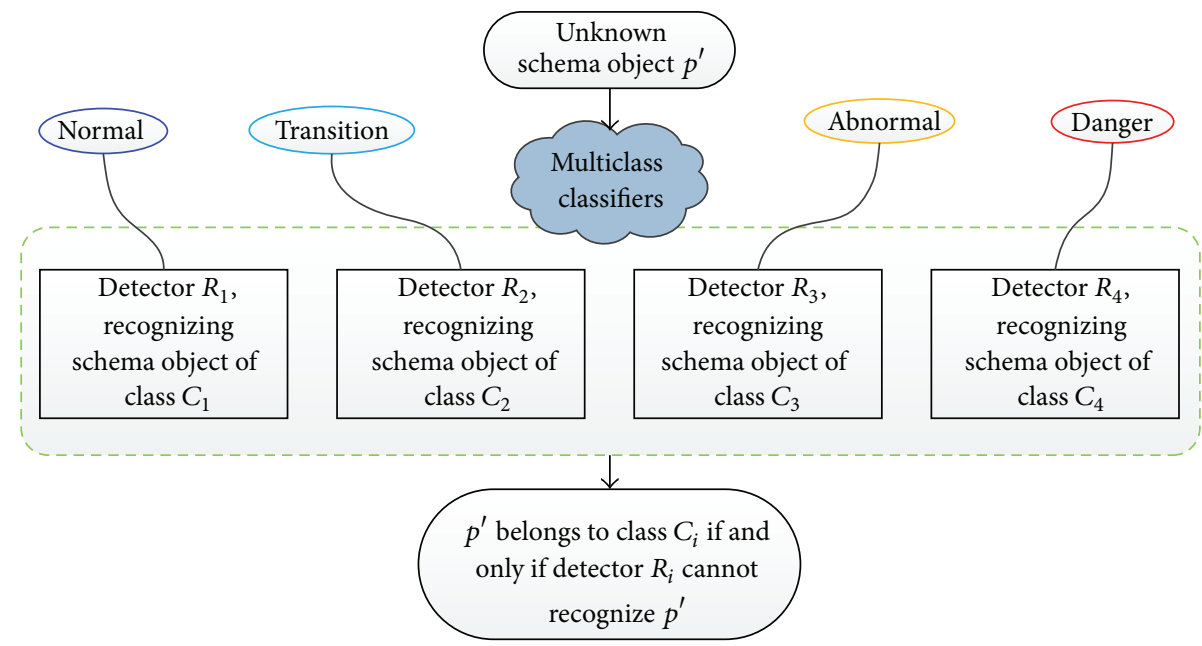

FIGURE 3: Immune classifier model of dynamic health assessment for shearer.

Definition 3. Receptor can recognize any one of the schema objects of a certain type. The degree of similarity between receptor and schema object can measure affinity:

$$
\begin{aligned}
\operatorname{affinity}(r, p) & =1-\frac{D(r, p)}{k} \\
D(r, p) & =\sqrt{\sum_{i=1}^{k}\left(r_{i}-p_{i}\right)^{2}},
\end{aligned}
$$

where $R$ is detectors set, $r \in R$.

$D(r, p)$ is the Euclidean distance of schema object $p$ and detector $r$.

The function value of affinity lies between 0 and 1 . The more similar the value between schema object $p$ and detector $r$, the greater the function value of affinity.

Definition 4. $\partial_{\text {selection }}$ is a choosing threshold for selected receptor and the value lies between 0 and 1 . The choosing threshold is very important, as it selects which receptors should be removed from detectors set in the step of training.

Definition 5. $\partial_{\text {detection }}$ is a testing threshold for detectors set. The value of testing threshold is the key to correctly classify detectors, as it decides which detectors should be activated in the step of testing.

3.4. Establishing the Assessment Model. In this section, the flows for establishing the dynamic health assessment model based on artificial immune algorithm are provided in detail. It mainly includes three steps.

3.4.1. Data Initialization. This step can be regarded as a data preprocessing stage. Each schema object is represented as a 9-dimensional vector $(p, c)=\left(p_{1}, p_{2}, \ldots, p_{9}, c\right), p \in P$. All object data of assessment indicators in the schema object set are normalized, so the attribute value is within the unit interval $[0,1]$ and $p_{i} \in(0,1)$. To correspond to the four patterns of shearer dynamic health assessment consequences, the training data of schema object set are divided into four types: normal mode $\left(D_{1}\right)$, transition mode $\left(D_{2}\right)$, abnormal mode $\left(D_{3}\right)$, and danger mode $\left(D_{4}\right)$.

3.4.2. Training Detectors Set. The purpose of training stage is generating an effective detector for each schema object. The steps for generating detector are given as follows.

Step 1. Take preprocessed $D_{i}$ as self-data set $D_{\text {self }}, D_{\text {self }}=$ $D_{i}$, so non-self-data set $D_{\text {nonself }}$ was made up by the other preprocessed data sets, $D_{\text {nonself }}=\left\{D_{1} \cup \cdots \cup D_{i-1} \cup D_{i+1} \cup\right.$ $\left.\cdots \cup D_{N}\right\}$. Initial detector $R_{i}$ is empty, $R_{i}=\emptyset$.

Step 2. Generate random alternative detectors set $R^{\prime}$.

Step 3. Calculate affinity between $R^{\prime}$ and $D_{\text {self }}$; if affinity $\left(p_{i}\right.$, $\left.r_{j}\right)>\partial_{\text {selection }}$, delete $r_{j}$ from $R^{\prime}$ (negative selection).

Step 4. Calculate affinity between $R^{\prime}$ and $D_{\text {nonself; if }}$ affinity $\left(p_{i}, r_{j}\right)<\partial_{\text {selection}}$, delete $r_{j}$ from $R^{\prime}$ (positive selection).

Step 5. Delete the individual from $D_{\text {nonself }}$, if it can be recognized by $R^{\prime}$.

Step 6. If $D_{\text {nonself }}=\emptyset$, detector $R_{i}$ is accomplished, and iteration is finished, $R_{i}=R_{i} \cup R^{\prime}$; otherwise, turn back to Step 2.

Using the same negative selection algorithm of generating detector, repeat calculation four times from class object $C_{1}$ to class object $C_{4}$ to all detector sets until every detector can distinguish self-class object and non-self-class object. The generation process of a detector is shown in Figure 4.

3.4.3. Testing Detectors Set. To distinguish self-class object and non-self-class object, all detectors are used for circulatory 


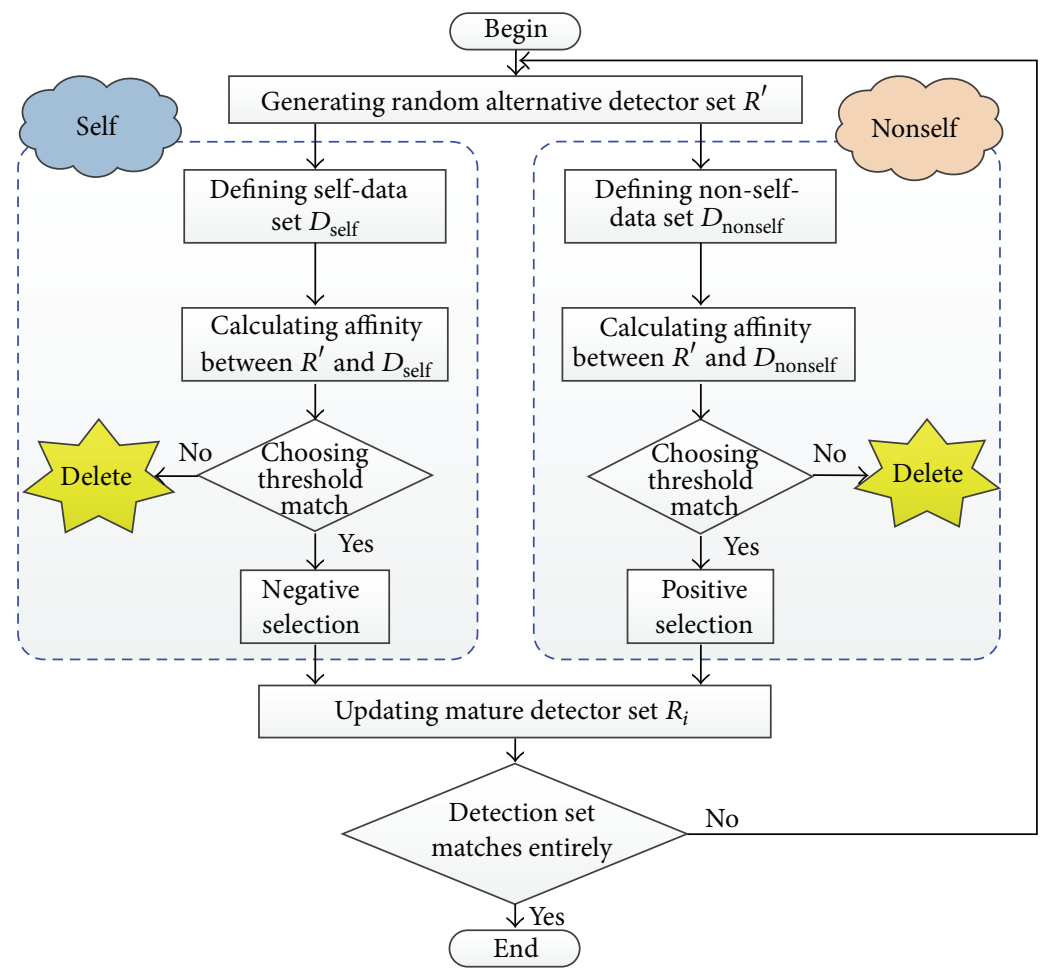

FIGURE 4: The generation process of a detector.

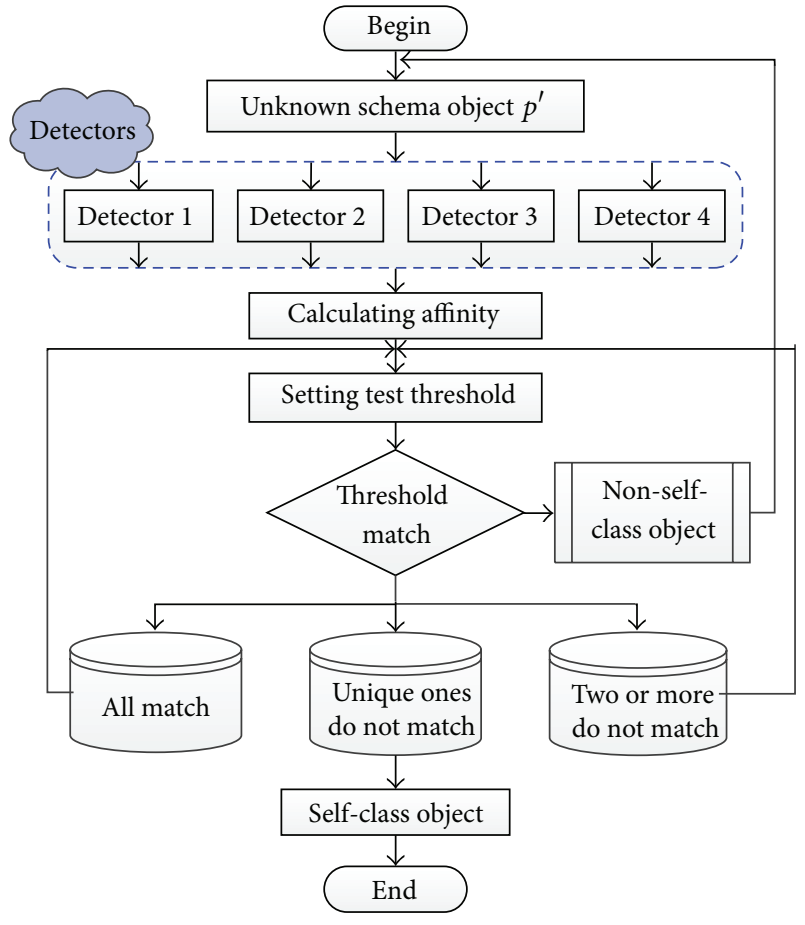

FIGURE 5: Flowchart of negative selection test for a new sample.

elimination for new sample in testing step. The flowchart of negative selection test for a new sample is shown in Figure 5. $p^{\prime}$ is sample data of unknown schema object for inputting. Then, calculate the value $\partial_{\text {detection }}$ between $p^{\prime}$ and all detectors $\left(R_{1} \sim R_{i}\right)$. If data $p^{\prime}$ can be recognized by detector $R_{m}(1 \leq$ $m \leq i)$, data $p^{\prime}$ does not belong to the schema object $C_{m}$. Repeat this process until all detectors are tested. The final consequence will be one of the following cases.

Case 1. If schema object $p^{\prime}$ only cannot be recognized by detector $R_{i}$, then schema object $p^{\prime}$ belongs to class object $C_{i}$.

Case 2. If schema object $p^{\prime}$ cannot be recognized by two or more detectors, set a new test threshold value. Calculate the value $\partial_{\text {detection }}$ between $p^{\prime}$ and nonactivated detectors. Repeat this process until only one detector remained nonactivated. Then, schema object $p^{\prime}$ belongs to the corresponding schema object of the last nonactivated detector.

Case 3. If schema object $p^{\prime}$ can be recognized by all detectors, set a new test threshold value. Calculate the value $\partial_{\text {detection }}$ between $p^{\prime}$ and all detectors. Repeat this process until only one detector remained nonactivated. Then, schema object $p^{\prime}$ belongs to the corresponding schema object of the last nonactivated detector.

\section{Simulation Examples and Application}

4.1. Simulation Examples. In this section, some simulation examples were put forward to verify the feasibility and efficiency of the proposed approach. 
TABLE 1: Normalized data of pattern objects for shearer.

\begin{tabular}{|c|c|c|c|c|c|c|c|c|c|c|}
\hline Number & $P_{1}$ & $P_{2}$ & $P_{3}$ & $P_{4}$ & $P_{5}$ & $P_{6}$ & $P_{7}$ & $P_{8}$ & $P_{9}$ & Categories \\
\hline 1 & 0.226 & 0.515 & 0.485 & 0.559 & 0.539 & 0.455 & 0.568 & 0.298 & 0.338 & $D_{1}$ \\
\hline 2 & 0.194 & 0.540 & 0.485 & 0.557 & 0.535 & 0.455 & 0.565 & 0.287 & 0.328 & $D_{1}$ \\
\hline 3 & 0.168 & 0.577 & 0.494 & 0.486 & 0.480 & 0.458 & 0.565 & 0.291 & 0.335 & $D_{1}$ \\
\hline 4 & 0.167 & 0.562 & 0.485 & 0.513 & 0.496 & 0.458 & 0.565 & 0.290 & 0.338 & $D_{1}$ \\
\hline$\vdots$ & $\vdots$ & $\vdots$ & $\vdots$ & $\vdots$ & $\vdots$ & $\vdots$ & $\vdots$ & $\vdots$ & $\vdots$ & $\vdots$ \\
\hline 185 & 0.257 & 0.509 & 0.494 & 0.461 & 0.489 & 0.458 & 0.565 & 0.296 & 0.343 & $D_{1}$ \\
\hline 186 & 0.258 & 0.519 & 0.506 & 0.471 & 0.497 & 0.455 & 0.565 & 0.295 & 0.343 & $D_{1}$ \\
\hline 187 & 0.000 & 0.485 & 0.497 & 0.444 & 0.432 & 0.458 & 0.570 & 0.309 & 0.329 & $D_{2}$ \\
\hline 188 & 0.227 & 0.503 & 0.488 & 0.417 & 0.422 & 0.458 & 0.568 & 0.281 & 0.327 & $D_{1}$ \\
\hline$\vdots$ & $\vdots$ & $\vdots$ & $\vdots$ & $\vdots$ & $\vdots$ & $\vdots$ & $\vdots$ & $\vdots$ & $\vdots$ & $\vdots$ \\
\hline 354 & 0.350 & 0.519 & 0.491 & 0.459 & 0.450 & 0.458 & 0.565 & 0.284 & 0.329 & $D_{1}$ \\
\hline 355 & 0.858 & 0.552 & 0.821 & 0.531 & 0.704 & 0.458 & 0.570 & 0.301 & 0.350 & $D_{3}$ \\
\hline 356 & 0.000 & 0.485 & 0.497 & 0.444 & 0.432 & 0.458 & 0.570 & 0.309 & 0.329 & $D_{2}$ \\
\hline 357 & 0.408 & 0.522 & 0.497 & 0.455 & 0.445 & 0.458 & 0.568 & 0.280 & 0.327 & $D_{1}$ \\
\hline : & $\vdots$ & $\vdots$ & $\vdots$ & $\vdots$ & $\vdots$ & $\vdots$ & $\vdots$ & $\vdots$ & $\vdots$ & $\vdots$ \\
\hline 587 & 0.000 & 0.485 & 0.497 & 0.444 & 0.432 & 0.458 & 0.570 & 0.309 & 0.329 & $D_{2}$ \\
\hline 588 & 0.773 & 0.556 & 0.990 & 0.659 & 0.653 & 0.458 & 0.568 & 0.304 & 0.348 & $D_{4}$ \\
\hline 589 & 0.850 & 0.503 & 0.907 & 0.630 & 0.619 & 0.458 & 0.568 & 0.301 & 0.348 & $D_{4}$ \\
\hline 590 & 0.854 & 0.540 & 0.861 & 0.587 & 0.635 & 0.458 & 0.568 & 0.303 & 0.349 & $D_{3}$ \\
\hline$\vdots$ & $\vdots$ & $\vdots$ & $\vdots$ & $\vdots$ & $\vdots$ & $\vdots$ & $\vdots$ & $\vdots$ & $\vdots$ & $\vdots$ \\
\hline 753 & 0.943 & 0.506 & 0.509 & 0.816 & 0.783 & 0.461 & 0.570 & 0.311 & 0.359 & $D_{3}$ \\
\hline 754 & 0.950 & 0.509 & 0.707 & 0.630 & 0.619 & 0.458 & 0.568 & 0.301 & 0.348 & $D_{4}$ \\
\hline 755 & 0.000 & 0.481 & 0.497 & 0.445 & 0.432 & 0.458 & 0.570 & 0.313 & 0.352 & $D_{2}$ \\
\hline 756 & 0.000 & 0.494 & 0.497 & 0.449 & 0.431 & 0.458 & 0.570 & 0.313 & 0.352 & $D_{2}$ \\
\hline$\vdots$ & $\vdots$ & $\vdots$ & $\vdots$ & $\vdots$ & $\vdots$ & $\vdots$ & $\vdots$ & $\vdots$ & $\vdots$ & $\vdots$ \\
\hline 893 & 0.042 & 0.491 & 0.497 & 0.386 & 0.329 & 0.458 & 0.570 & 0.294 & 0.339 & $D_{2}$ \\
\hline 894 & 0.151 & 0.494 & 0.491 & 0.405 & 0.419 & 0.458 & 0.570 & 0.290 & 0.342 & $D_{2}$ \\
\hline 895 & 0.950 & 0.494 & 0.503 & 0.708 & 0.724 & 0.461 & 0.570 & 0.296 & 0.354 & $D_{3}$ \\
\hline 896 & 0.399 & 0.488 & 0.491 & 0.389 & 0.393 & 0.458 & 0.570 & 0.288 & 0.338 & $D_{2}$ \\
\hline$\vdots$ & $\vdots$ & $\vdots$ & $\vdots$ & $\vdots$ & $\vdots$ & $\vdots$ & $\vdots$ & $\vdots$ & $\vdots$ & $\vdots$ \\
\hline 1072 & 0.950 & 0.506 & 0.506 & 0.621 & 0.783 & 0.461 & 0.570 & 0.297 & 0.358 & $D_{3}$ \\
\hline 1073 & 0.948 & 0.506 & 0.500 & 0.695 & 0.658 & 0.461 & 0.570 & 0.298 & 0.358 & $D_{3}$ \\
\hline 1074 & 0.950 & 0.509 & 0.707 & 0.630 & 0.619 & 0.458 & 0.568 & 0.301 & 0.348 & $D_{4}$ \\
\hline 1075 & 0.854 & 0.506 & 0.920 & 0.587 & 0.635 & 0.458 & 0.568 & 0.303 & 0.349 & $D_{4}$ \\
\hline$\vdots$ & $\vdots$ & $\vdots$ & $\vdots$ & $\vdots$ & $\vdots$ & $\vdots$ & $\vdots$ & $\vdots$ & $\vdots$ & $\vdots$ \\
\hline 1200 & 0.347 & 0.500 & 0.475 & 0.535 & 0.534 & 0.464 & 0.527 & 0.298 & 0.326 & $D_{1}$ \\
\hline
\end{tabular}

The sample data were acquired from the shearer in 22210 fully mechanized coal face of Zhong Ping Energy Chemical Group No. 6 Mine. Depending on the assessment model of prediction approach of shearer dynamic health assessment, the acquired data were normalized so that the object data were represented as a 9-dimensional vector, $(p, c)=\left(p_{1}, p_{2}, \ldots, p_{9}, c\right), p \in P$. The data in the schema object set was initialized, so the attribute value was within the unit interval $[0,1], p_{i} \in(0,1)$. The training data of schema object set were divided into four types: normal mode $\left(D_{1}\right)$, transition mode $\left(D_{2}\right)$, abnormal mode $\left(D_{3}\right)$, and danger mode $\left(D_{4}\right)$. As shown in Table 1, 1000 groups of data were 


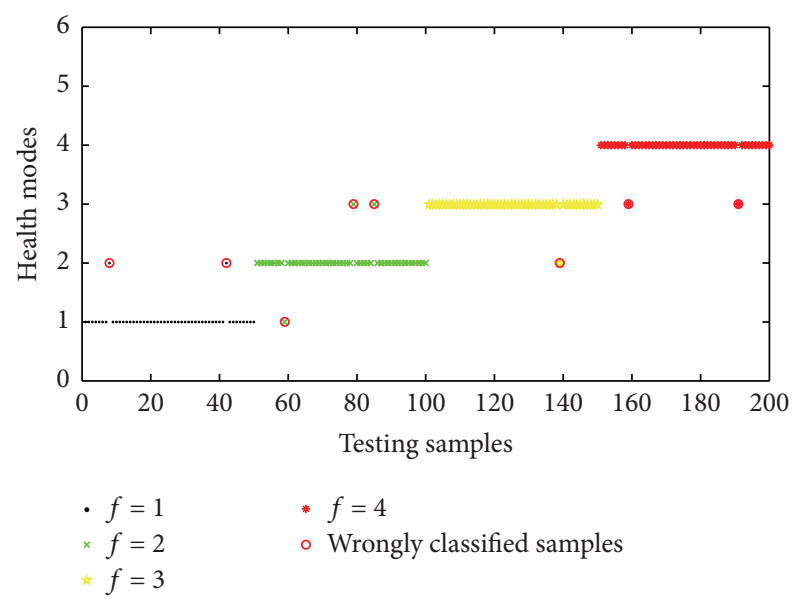

Figure 6: Classification results of the classifier based on artificial immune algorithm.

randomly chosen to train the detectors set and the last 200 groups were used to test the classification performance of the trained detectors.

After the assessment model based on artificial immune algorithm was trained, the multiclass classifiers of the assessment system were constituted by four detectors, and each detector only could not recognize corresponding class object of particular assessment consequence mode. Actually, if the input schema object only could not be recognized by one detector, then the schema object belongs to this class object.

After the training phase, an assessment system could be obtained. In order to verify the accuracy of the model, the remaining 200 samples were utilized to test its performance. The prediction consequence was given as in Figure 6. As shown in Figure 6, only eight testing samples were misclassified and circled in red. The ordinate values $1,2,3$, and 4 corresponded to four assessment consequences of shearer dynamic health modes, and identification accuracies of four detectors were $96 \%, 94 \%, 98 \%$, and $96 \%$, respectively. The overall average classification accuracy was $96 \%$, which satisfied the engineering requirement. The testing results indicated that the proposed approach performed with lower deviation and could be applied in the assessment of shearer dynamic health.

In order to indicate the meliority of assessment model based on artificial immune algorithm, the assessment model based on back propagation-neural network (BP-NN) and support vector machine (SVM) was provided to solve the problem of the above example. The training samples and testing samples were the same. The configurations of simulation environment for three algorithms were uniform and the relevant parameters were in common with the above example. The prediction consequence of the assessment model based on BP-NN was given as in Figure 7. As shown in Figure 7, twenty-one testing samples were misclassified and some samples had a large deviation to real situations. The classification accuracies for four health modes were $90 \%, 86 \%, 90 \%$, and $92 \%$, and overall average classification accuracy was $89.5 \%$.

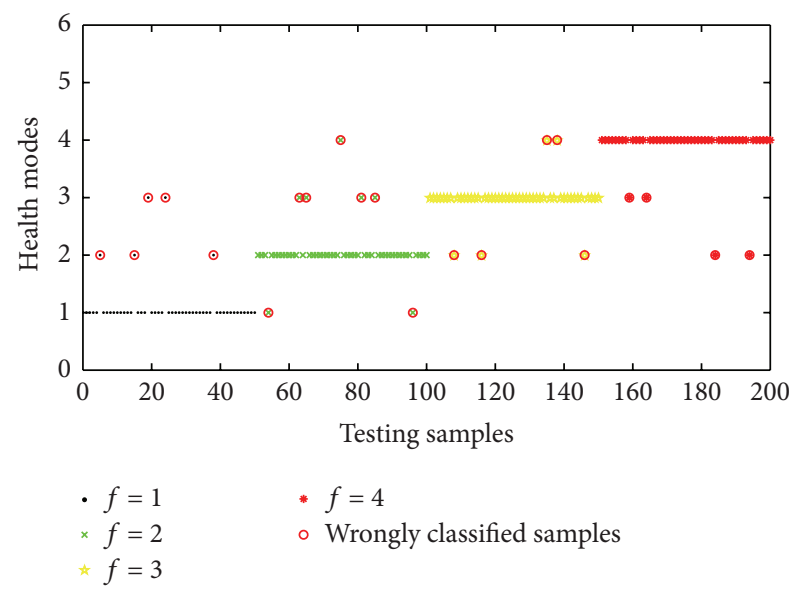

FIGURE 7: Classification results of the classifier based on BP-NN.

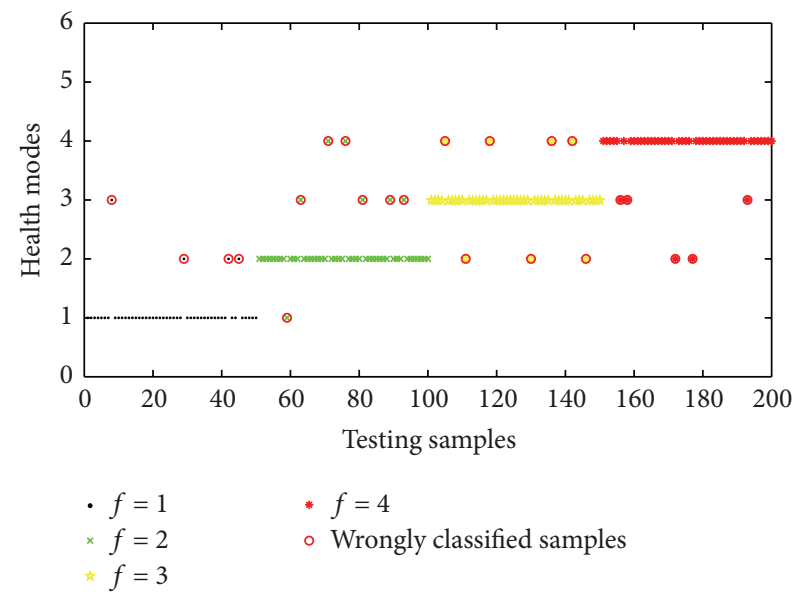

FIGURE 8: Classification results of the classifier based on SVM.

The prediction consequence of the assessment model based on SVM was given as in Figure 8. As shown in Figure 8 , twenty-three testing samples were misclassified and some samples had a large deviation to real situations. The classification accuracies for four health modes were $92 \%, 86 \%, 88 \%$, and $90 \%$, and overall average classification accuracy was $88.5 \%$. It was observed that the proposed method had a better classification capability and performance than the competing methods. With the benefits of artificial immune algorithm in uncertain fields, the proposed classifier could obtain higher classification accuracy than single BP$\mathrm{NN}$ and SVM classifiers.

4.2. Further Discussion. In order to further compare and analyze the overall performance of SVM, BP-NN, and AI, the same 1200 samples are experimented with. In this example, a certain number of samples, denoted by training size $\left(T_{\text {size }}\right)$, are randomly selected from the data as the training samples and 200 samples are randomly selected from the remaining $1200-T_{\text {size }}$ samples as the testing samples. Each learning algorithm is then trained and tested 200 times and the classification error rate is recorded as the final result. In this 


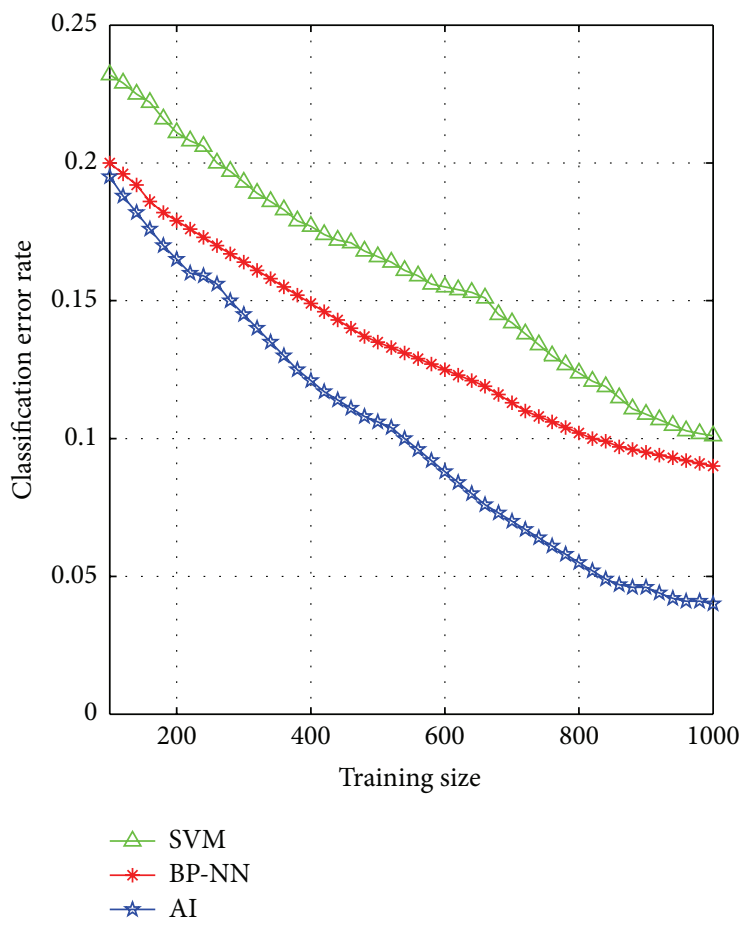

FIGURE 9: The changes of classification error rate with different training sizes.

study, the training size of the example varies over $T_{\text {size }}=$ $100,120,140,160, \ldots, 1000$. That is to say, we run several trials over the algorithms with training size ranging from 100 to 1000 . The classification error rate $m / n$ (where $m$ is the classification error times and $n$ is the total test times) is chosen as the metric to express the result as a proportion of the optimal solution.

Figure 9 plots the means of this metric (classification error rate) for each trial as a function of problem size $T_{\text {size }}$. It can be seen that for all trials the classification error rate decreases nonlinearly with $T_{\text {size }}$ and the classification accuracy of AI outperforms BP-NN and SVM for all $T_{\text {size }}$.

From Figure 9, it is obvious that the classification error rate descent velocity of $\mathrm{AI}$ is the fastest across different training sizes and owns stronger generalization ability than BP$\mathrm{NN}$ and SVM regardless of the training size. What is more, the classification accuracy of AI is more supernal and robust. Therefore, the AI algorithm can obtain a relatively high accuracy to provide an effective support tool for dynamic health assessment for shearer.

4.3. Industrial Application. In this section, a system based on the proposed approach had been developed and applied in the field of shearer dynamic health assessment as shown in Figure 10.

As Figure 10 showed, the "gateway controller" and "ground monitoring center" were used to control and monitor the shearer running parameters. The system based on the proposed approach was uploaded into the gateway controller. The pulling speed, the left cutting motor current, the right cutting motor current, the left pulling motor current, the right pulling motor current, the left cutting motor temperature, the right cutting motor temperature, the left pulling motor temperature, and the right pulling motor temperature were collected every $1 \mathrm{~Hz}$ from the shearer controller and the collected data were transmitted to the gateway controller. Then, the changes of shearer dynamic health assessment consequence were identified and showed on "monitoring interface for shearer dynamic health assessment."

In order to illustrate the application effect of the proposed approach, the shearer was running in fully mechanized coal face from $135.0 \mathrm{~m}$ to $150.0 \mathrm{~m}$ by the manual operation. The dynamic health assessment curve based on the proposed classifier was shown in Figure 11, and the ordinate values of $1,2,3$, and 4 denoted four health classes: normal mode, transition mode, abnormal mode, and danger mode. The curve showed two obvious changes in segments A and $\mathrm{B}$. The ordinate values were leaped from 1 to 3 , which means that the shearer health status had a sudden change from normal mode to abnormal mode. The changes of some operational parameters were plotted in Figure 12. The cutting motor current had a noticeable increase when the right cutting drum cut the floor between $135.0 \mathrm{~m}$ and $138.5 \mathrm{~m}$ and the left cutting drum cut the roof between $146.0 \mathrm{~m}$ and $148.0 \mathrm{~m}$. The results of shearer dynamic health assessment based on the proposed system were almost completely consistent with the actual cutting status of shearer.

\section{Conclusions and Future Work}

The main contribution of this paper was that a methodology based on artificial immune algorithm for the assessment of 

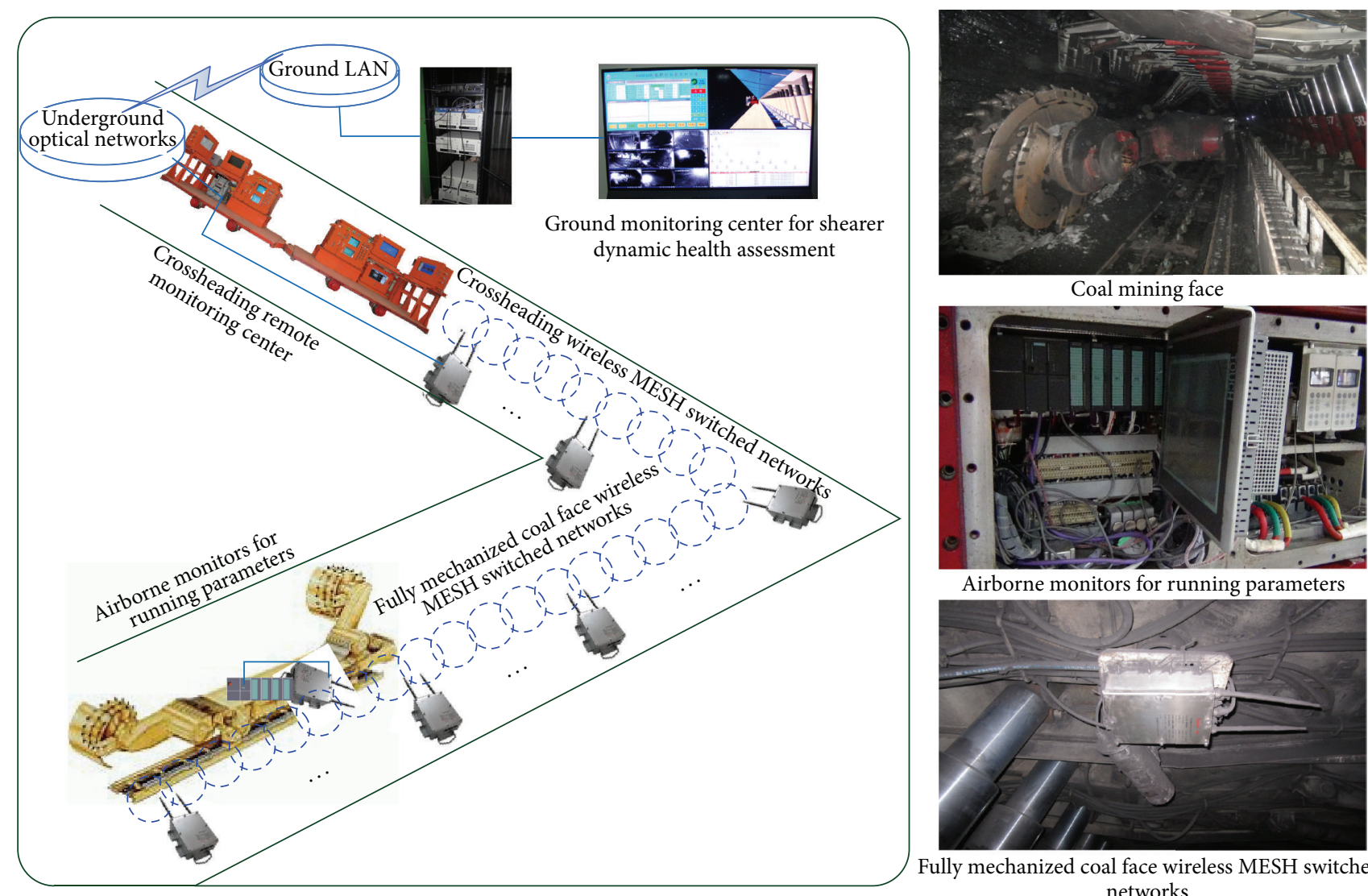

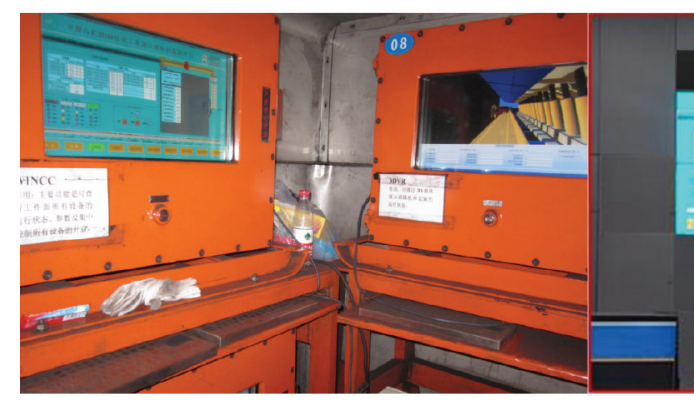

Gateway controller

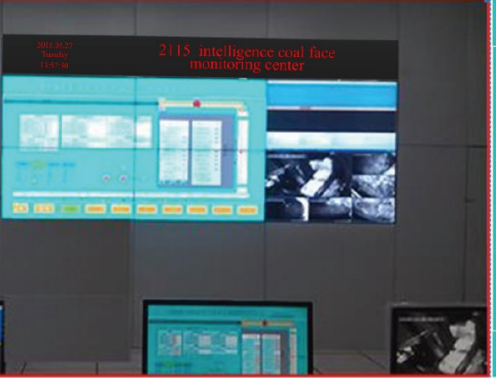

Ground monitoring center

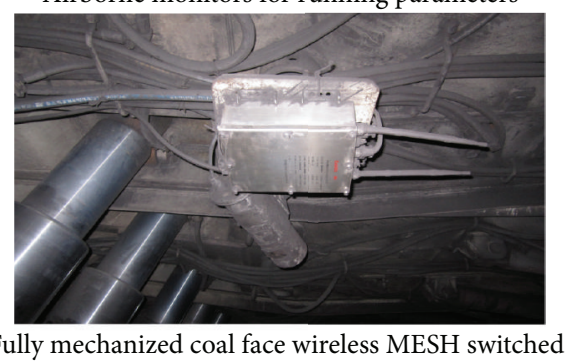
networks

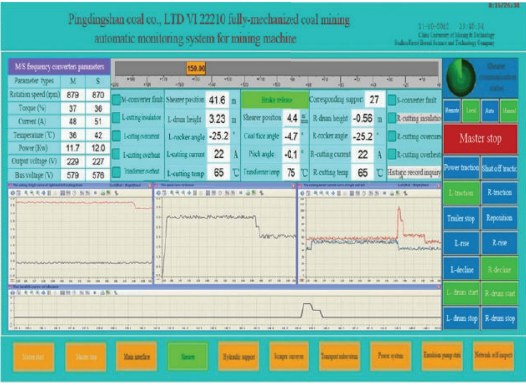

Monitoring interface for shearer dynamic health assessment

FIGURE 10: Hardware construction in fully mechanized coal face.

shearer dynamic health status was presented. The detailed flows for the proposed approach were described, including three critical steps, that is, assessment indicators selecting, data acquisition and initialization, and multiclass classifiers training and testing. In order to verify the feasibility and efficiency of the proposed approach, a simulation example was provided and some comparisons with other algorithms were carried out. The simulation results showed that the proposed approach was outperforming others. Finally, the proposed approach was applied to an engineering problem of shearer dynamic health assessment. The industrial application results showed that the paper research achievements could be used combining with shearer automation control system in fully mechanized coal face and had obvious effectiveness on reducing operating trouble and production accident of shearer and improving coal production efficiency further. The artificial immune algorithm could obtain a relatively high accuracy to provide an effective support tool for dynamic health assessment for shearer.

In future studies, the authors plan to investigate some improvements for the proposed approach. Possible improvements may include the combination of artificial immune algorithm with other intelligent algorithms for better performance. In addition, the applications of the proposed approach in dynamic health assessment domain are worth further study from the authors. 


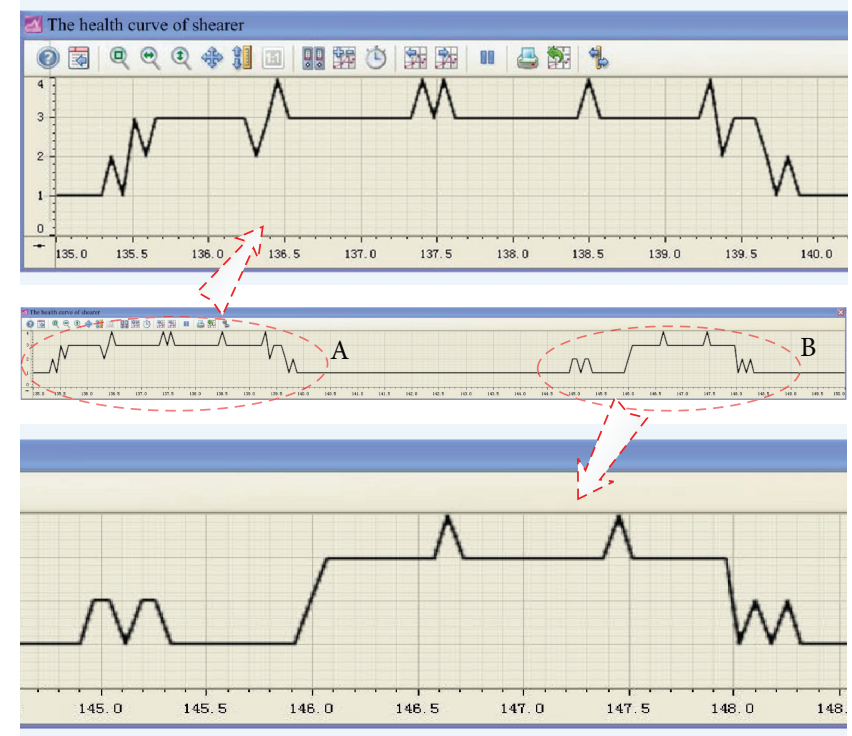

FigURE 11: The dynamic health assessment curve of shearer based on the proposed system.

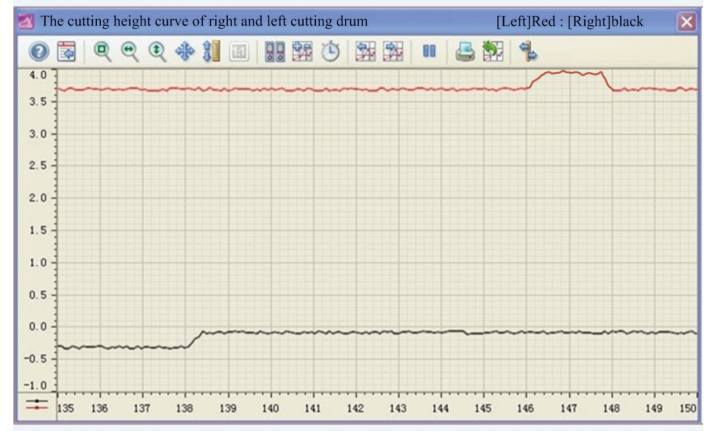

The cutting height curve of right and left cutting drum

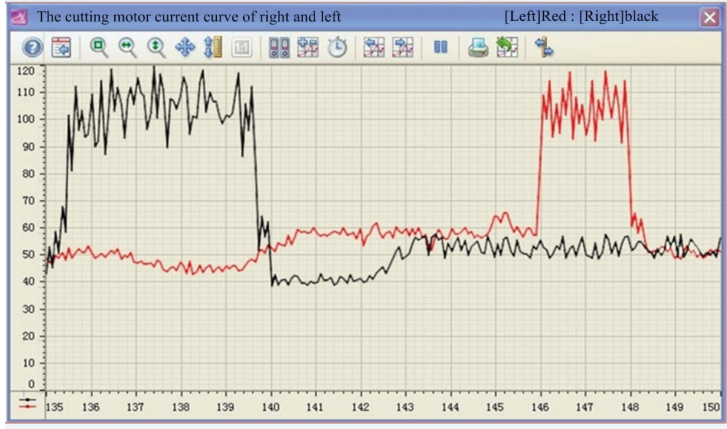

The right and left cutting motors current curve

FIGURE 12: The operational parameters curve of shearer with the manual operation.

\section{Competing Interests}

The authors declare that there is no conflict of interests regarding the publication of this paper.

\section{Acknowledgments}

The support of Joint Funds of the National Natural Science Foundation of China (no. U1510117), National Key Basic Research Program of China: Key Fundamental Research on the Unmanned Mining Equipment in Deep Dangerous Coal Bed (no. 2014CB046301), the Priority Academic Program Development (PAPD) of Jiangsu Higher Education Institutions, and the Innovation Funds of Production and Research Cooperation Project in Jiangsu Province (BY2014107) in carrying out this research is gratefully acknowledged.

\section{References}

[1] B. H. G. Brady and E. T. Brown, "Longwall and caving mining methods," in Rock Mechanics: For Underground Mining, pp. 430-483, Springer, Amsterdam, The Netherlands, 2006.

[2] X. Zhang, X. Ma M, and Z. S. Yang, "Analysis and diagnosis of coal shearer machine fault based on improved support vector theory," in Proceedings of the International Conference on Electrical, Automation and Mechanical Engineering, Phuket, Thailand, July 2015.

[3] F. Gao, L. J. Xiao, W. Y. Zhong, and W. Liu, "Fault diagnosis of shearer based on fuzzy inference," Applied Mechanics and Materials, vol. 52-54, pp. 1577-1580, 2011.

[4] S. Kerroumi, X. Chiementin, and L. Rasolofondraibe, "Dynamic classification method of fault indicators for bearings' monitoring," Mechanics and Industry, vol. 14, no. 2, pp. 115-120, 2013. 
[5] Z.- W. He, M.-Y. Gao, G.-J. Ma, Y.-Y. Liu, and S.-X. Chen, "Online state-of-health estimation of lithium-ion batteries using Dynamic Bayesian Networks," Journal of Power Sources, vol. 267, pp. 576-583, 2014.

[6] F.-R. Liu, Q.-L. Wang, and X.-Z. Gao, "Survey of artificial immune system," in Proceedings of the 1st International Symposium on Systems and Control in Aerospace and Astronautics (ISSCAA '06), p. 989, 2006.

[7] K.-J. Wang, K.-H. Chen, and M.-A. Angelia, "An improved artificial immune recognition system with the opposite sign test for feature selection," Knowledge-Based Systems, vol. 71, pp. 126$145,2014$.

[8] I. Jenhani and Z. Elouedi, "Re-visiting the artificial immune recognition system: a survey and an improved version," Artificial Intelligence Review, vol. 42, no. 4, pp. 821-833, 2012.

[9] W. Zhong-Bin, N. Wen-Feng, and L. Shu-Bin, "Research on key technologies of remote monitoring platform for shearer," in Proceedings of the International Conference on IEEE Measuring Technology and Mechatronics Automation (ICMTMA '09), vol. 1, pp. 316-319, Zhangjiajie, China, April 2009.

[10] X. Zhou, Z. Wang, C. Tan, R. Ji, and X. Liu, "A novel approach for shearer memory cutting based on fuzzy optimization method," Advances in Mechanical Engineering, vol. 5, Article ID 319272, 2013.

[11] P. W. Tse and Y. L. Tse, "On-road mobile phone based automobile safety system with emphasis on engine health evaluation and expert advice," in Proceedings of the Technology Management for Emerging Technologies Conference (PICMET '12), pp. 3232-3241, IEEE, Vancouver, Canada, July 2012.

[12] D. Black and A. K. Winiewicz, "Internal network device dynamic health monitoring," US Patent 7143153, 2006.

[13] N. M. Vichare and M. G. Pecht, "Prognostics and health management of electronics," IEEE Transactions on Components and Packaging Technologies, vol. 29, no. 1, pp. 222-229, 2006.

[14] M. Pecht and R. Jaai, "A prognostics and health management roadmap for information and electronics-rich systems," Microelectronics Reliability, vol. 50, no. 3, pp. 317-323, 2010.

[15] J.-J. Yang, R. Xi, D. Liu, H. Jiang, and M. Wu, "Analysis of shearer gear vibration in the no-load state," in Proceedings of the 5th Conference on Measuring Technology and Mechatronics Automation (ICMTMA '13), pp. 247-250, IEEE, Hong Kong, January 2013.

[16] X.-D. Yin, A.-G. Liu, X.-M. Dong, and W.-S. Hao, "Study on health evaluation system for coal mine speed reducer based on embedded system," Applied Mechanics and Materials, vol. 105, pp. 660-663, 2012.

[17] D. Mascareñas, C. Plont, C. Brown et al., "A vibro-haptic human-machine interface for structural health monitoring," Structural Health Monitoring, vol. 13, no. 6, pp. 671-685, 2014.

[18] F. Cerda, S. Chen, J. Bielak, J. H. Garrett, P. Rizzo, and J. Kovačević, "Indirect structural health monitoring of a simplified laboratory-scale bridge model," Smart Structures and Systems, vol. 13, no. 5, pp. 849-868, 2014.

[19] J. F. Zubizarreta-Rodriguez and S. Vasudevan, "Condition monitoring of brushless DC motors with non-stationary dynamic conditions," in Proceedings of the IEEE International Instrumentation and Measurement Technology Conference (I2MTC '14), pp. 62-67, IEEE, Montevideo, Uruguay, May 2014.

[20] S. Herrmann, J. Wellnitz, S. Jahn, and S. Leonhardt, "Structural health monitoring for carbon fiber resin composite car body structures," in Sustainable Automotive Technologies 2013, pp. 7596, Springer, 2014.
[21] J. D. Farmer, N. H. Packard, and A. S. Perelson, "The immune system, adaptation, and machine learning," Physica D: Nonlinear Phenomena, vol. 22, no. 1-3, pp. 187-204, 1986.

[22] A. Ishiguro, Y. Shirai, T. Kondo, and Y. Uchikawa, "Immunoid: an architecture for behavior arbitration based on the immune networks," in Proceedings of the IEEE/RSJ International Conference on Intelligent Robots and Systems (IROS '96), vol. 3, pp. 1730-1738, November 1996.

[23] Z. Tang, T. Yamaguchi, K. Tashima, O. Ishizuka, and K. Tanno, "Multiple-valued immune network model and its simulations," in Proceedings of the 27th International Symposium on IEEE Multiple-Valued Logic, pp. 233-238, Antigonish, Canada, May 1997.

[24] F. Abbattista, G. Di Gioia, G. Di Santo, and A. M. Fanelli, "An associative memory based on the immune networks," in Proceedings of the IEEE International Conference on Neural Networks (ICNN '96), vol. 1, pp. 519-523, 1996.

[25] Z.-L. Deng, G.-Z. Tan, P. He, and J.-X. Ye, "A fuzzy logic resource allocation and memory cell pruning based artificial immune recognition system," Journal of Central South University, vol. 21, no. 2, pp. 610-617, 2014.

[26] L. N. De Castro and F. J. Von Zuben, "Learning and optimization using the clonal selection principle," IEEE Transactions on Evolutionary Computation, vol. 6, no. 3, pp. 239-251, 2002.

[27] J.-S. Chun, M.-K. Kim, H.-K. Jung, and S.-K. Hong, "Shape optimization of electromagnetic devices using immune algorithm," IEEE Transactions on Magnetics, vol. 33, no. 2, pp. 1876-1879, 1997.

[28] S. Endoh, N. Toma, and K. Yamada, "Immune algorithm for nTSP," in Proceedings of the 1998 IEEE International Conference on Systems, Man, and Cybernetics, vol. 4, pp. 3844-3849, October 1998.

[29] A. Ishiguro, T. Kondo, Y. Watanabe, and Y. Uchikawa, "Dynamic behavior arbitration of autonomous mobile robots using immune networks," in Proceedings of the IEEE International Conference on Evolutionary Computation, vol. 2, pp. 722-727, Perth, Wash, USA, November 1995.

[30] P. K. Harmer, P. D. Williams, G. H. Gunsch, and G. B. Lamont, "An artificial immune system architecture for computer security applications," IEEE Transactions on Evolutionary Computation, vol. 6, no. 3, pp. 252-280, 2002.

[31] D.-F. Pan, M.-G. Wang, Y.-N. Zhu, and K. Han, "An optimization algorithm for locomotive secondary spring load adjustment based on artificial immune," Journal of Central South University, vol. 20, no. 12, pp. 3497-3503, 2013.

[32] S. S. F. Souza, R. Romero, and J. F. Franco, "Artificial immune networks Copt-aiNet and Opt-aiNet applied to the reconfiguration problem of radial electrical distribution systems," Electric Power Systems Research, vol. 119, pp. 304-312, 2015.

[33] R.-L. Zhang, M.-Y. Shan, X.-H. Liu, and L.-H. Zhang, "A novel fuzzy hybrid quantum artificial immune clustering algorithm based on cloud model," Engineering Applications of Artificial Intelligence, vol. 35, pp. 1-13, 2014.

[34] P. Savsani, R. L. Jhala, and V. Savsani, "Effect of hybridizing Biogeography-Based Optimization (BBO) technique with Artificial Immune Algorithm (AIA) and Ant Colony Optimization (ACO)," Applied Soft Computing Journal, vol. 21, pp. 542-553, 2014.

[35] R. J. Kuo, S. S. Chen, W. C. Cheng, and C. Y. Tsai, "Integration of artificial immune network and K-means for cluster analysis," Knowledge and Information Systems, vol. 40, no. 3, pp. 541-557, 2014. 

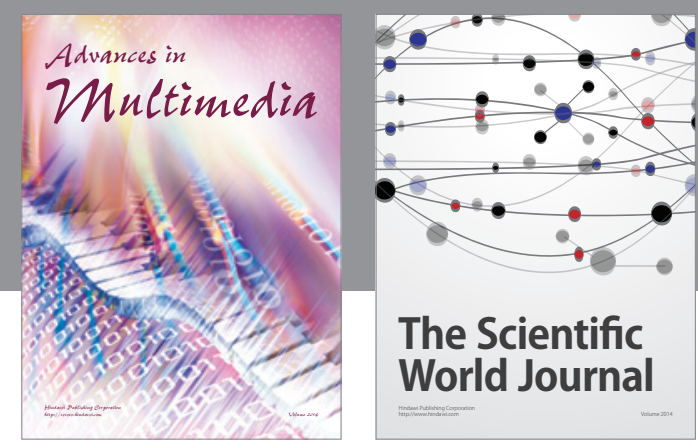

The Scientific World Journal
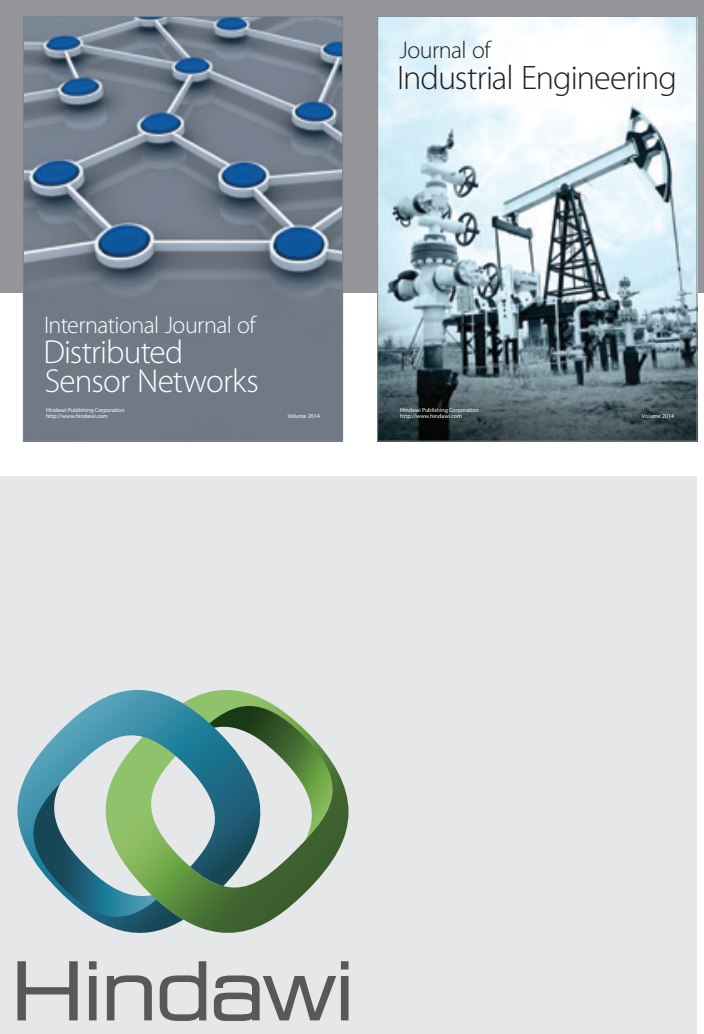

Submit your manuscripts at

http://www.hindawi.com

\section{Computer Networks} and Communications
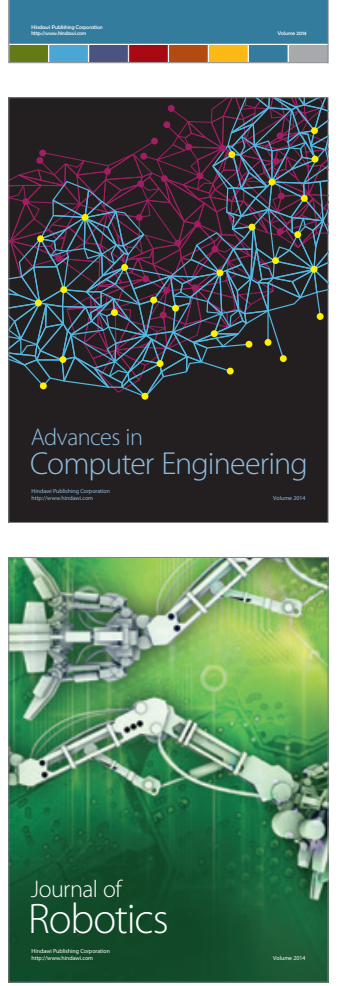
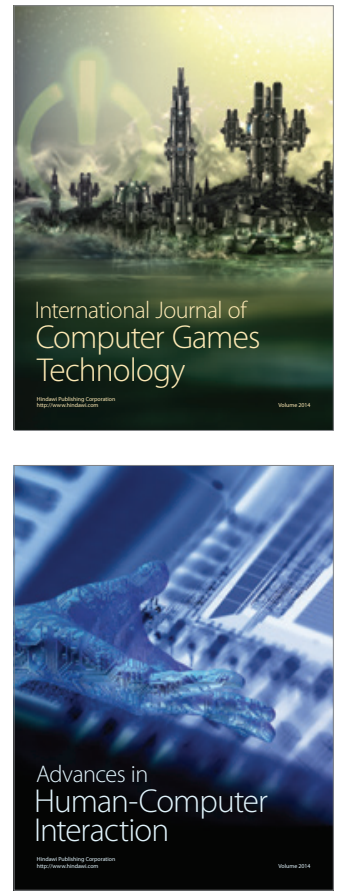
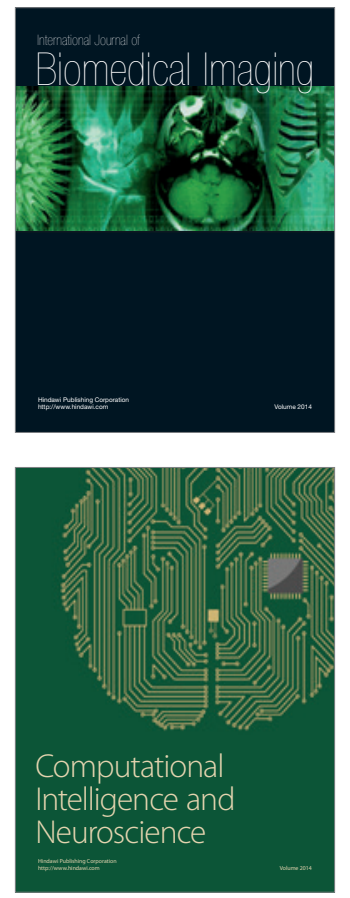
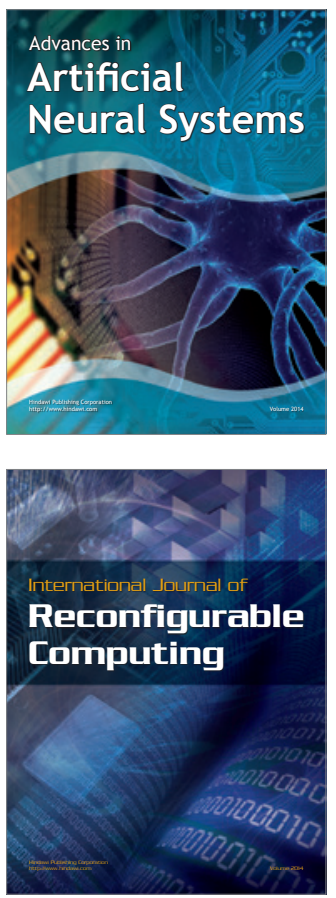
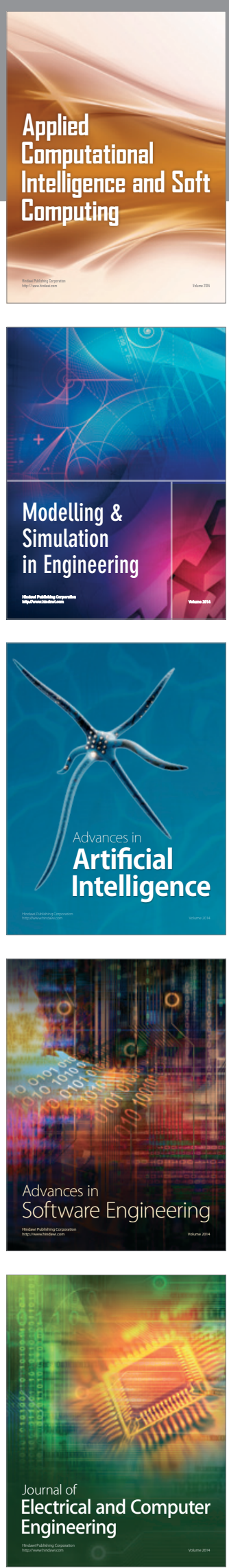